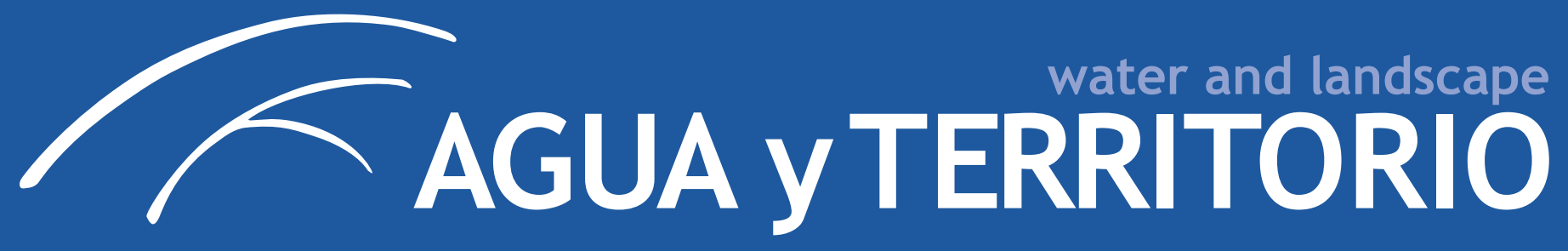

\title{
¿Está relacionada la eficiencia en el servicio urbano de agua con la titularidad del gestor?
}

\author{
Is Efficiency in Urban Water Services related to the Suppliers' Public \\ or Private Ownership of the Resource? \\ Marta Suárez-Varela Maciá
}

Universidad de Valencia. Valencia, España. marcesua@uv.es

\begin{abstract}
Resumen - El presente estudio tiene como objetivo realizar una revisión de la literatura acerca de qué forma de titularidad de los servicios de agua urbana, pública o privada, se muestra superior en términos de eficiencia. Con el fin de arrojar luz sobre esta cuestión hemos realizado, en primer lugar, un análisis de la situación de la industria a nivel internacional. A continuación se ofrece una exposición de los principales argumentos a favor y en contra de la privatización para finalizar con el examen de la evidencia empírica al respecto. La revisión efectuada no nos permite concluir que alguna de las formas de gestión se muestre indiscutiblemente superior en términos de eficiencia. Sin embargo, es posible detectar a la vista de la misma algunos desafíos y recomendaciones para futuras investigaciones.

Abstract - The present study aims to provide an overview of the available literature on the effects of public and private ownership on the efficiency of urban water suppliers. In order to shed more light on this issue, in the first place we offer an analysis of the industry's current conditions at an international level. Next, we examine the main arguments for and against privatization of water suppliers, followed by a comprehensive survey of the empirical evidence in that regard. Based on that review we conclude that neither public nor private types of water company ownership models have demonstrated to be irrefutably superior in terms of efficiency. However, we point out some of the challenges and offer recommendations for future research.
\end{abstract} Palabras clave: gestión del agua; empresas de agua; eficiencia; titularidad del gestor
Keywords: water management; water utilities; efficiency; ownership

Información Artículo: Recibido: 19 enero $2015 \quad$ Revisado: 30 junio 2015

Aceptado: 26 julio 2015

Códigos JEL: L95 Q25

( ) Universidad de Jaén / Seminario Permanente Agua, Territorio y Medio Ambiente (CSIC) 


\section{INTRODUCCIÓN}

El agua es un recurso escaso e imprescindible para la vida que cuenta con una serie de características que la convierten en objeto de especial atención por parte de los economistas. En primer lugar, se trata de un bien de mérito que sirve a objetivos económicos, medioambientales y de desarrollo social ${ }^{1}$ y que genera, también, importantes externalidades. Estas características, unidas a la condición del agua de satisfacer una necesidad básica humana, comporta la necesidad de ejercer una mayor supervisión por parte del estado y los agentes sociales, de forma que se garantice el acceso universal y una adecuada provisión, así como una óptima distribución del recurso entre distintos usos rivales como la industria, agricultura, hogares y ecosistemas².

De otro lado, el agua es un bien económico que presenta simultáneamente rasgos de bien público y privado. El agua, considerada como recurso natural, es un bien que satisface las propiedades de no rivalidad y exclusión en el consumo inherentes a los bienes públicos (al menos hasta un determinado nivel de consumo en el que el recurso comienza a ser escaso y los beneficios marginales que proporciona a la comunidad comienzan a decrecer); sin embargo, el hecho de que el servicio de acceso al agua deba ser provisto a través de una red de infraestructuras implica que la cantidad disponible del recurso se limite a la que puede ser servida por la red (rivalidad) y, también, que se pueda excluir de su consumo a determinados individuos, confiriéndole, por tanto, algunos de los rasgos de bien privado. Estamos, en consecuencia, ante un bien público impuro, cuya provisión es a menudo desaconsejable delegar a las fuerzas competitivas del mercado.

El debate acerca de qué forma de titularidad de la gestión de los servicios de agua, pública o privada, se muestra superior, ha sido recurrente durante las últimas tres décadas. En particular, uno de los argumentos más habitualmente esgrimidos a favor de la privatización es la mejora de la eficiencia. Son muchos los beneficios que cabe esperar de una gestión eficiente de los servicios de agua. Desde el punto de vista económico y social, una mejora en la gestión tiende a producir una disminución de los costes, repercutiendo consecuentemente en las tarifas pagadas por el usuario final. De esta forma pueden generarse importantes externalidades positivas para la sociedad, como facilitar el acceso universal al recurso o disminuir los costes soportados por aquellas industrias y sectores que utilizan el agua como input en su proceso de producción, pudiendo incluso mejorar en última instancia la competitividad de la economía. De otro lado, una gestión eficiente de los servicios de agua contribuye a minimizar el consumo de recursos como el agua extraída del medio, la energía, reactivos y otros materiales, reduciendo el correspondiente impacto negativo en el medio ambiente.

En este orden de ideas, la búsqueda de la eficiencia en la gestión de un servicio público como el servicio urbano de agua se revela como un factor de vital trascendencia para el desarrollo económico y social y la mejora de la sostenibilidad medioambiental, más si cabe en el contexto de crisis económica en el que nos

\footnotetext{
1 OECD, 2003.

2 Idem.
}

encontramos actualmente. Esto ha suscitado que en los últimos años la investigación haya puesto un especial foco de atención en identificar cuáles son las fórmulas de gestión que garantizan una mayor eficiencia.

Este artículo se propone realizar una revisión de la literatura teórica y empírica existente hasta la fecha para contribuir al debate sobre qué forma de titularidad de los servicios de agua urbana resulta más adecuada desde la perspectiva de la eficiencia en la gestión. Para ello, en primer lugar, vamos a hablar de las características de la industria del agua y su situación internacional. En el segundo apartado, trataremos los factores que desde el punto de vista teórico se han ofrecido a favor y en contra de la privatización para, en tercer lugar, realizar un análisis de la literatura al respecto. Finalmente, en la última sección se recogerán las conclusiones y recomendaciones para investigaciones futuras.

\section{Características de la INDUSTRIA DEL AGUA Y SITUACIÓN INTERNACIONAL}

La industria del agua presenta una serie de características particulares que la diferencian de otras actividades productivas y condicionan su estructura. Se trata de un sector intensivo en el factor capital y elevados costes fijos, que se caracteriza además por la necesidad de una alta inversión inicial, la existencia de economías de escala y de gama. En consecuencia, la industria del agua se configura como un monopolio natural.

Los servicios urbanos de agua son provistos a través del denominado ciclo integral del agua. En una primera fase, componen este ciclo una serie de actividades que incluyen la captación del recurso hídrico, así como su potabilización y transporte hasta el núcleo urbano para su posterior distribución a los hogares a través de una red urbana de tuberías. La segunda fase comprende la recogida de las aguas residuales a través de una red de alcantarillado, el transporte de las mismas y su depuración antes de ser vertidas de nuevo al medio ambiente. En cuanto al nivel de gobierno encargado de la adecuada provisión de estos servicios, en la mayoría de los países son los gobiernos locales, por ser el nivel de administración pública más cercano al ciudadano, los responsables últimos de la provisión de los servicios públicos, entre ellos los servicios de agua.

Como hemos comentado anteriormente, el agua es una necesidad básica, por lo que su adecuada provisión y acceso universal es objeto de estrecha vigilancia por parte de los poderes públicos. Según la Asociación Mundial para el Agua ${ }^{3}$, el ciclo integral de la gestión del agua ha sido definido como "un proceso que promueve el desarrollo y la gestión coordinada del agua, la tierra y los recursos relacionados con estos, con el fin de maximizar el bienestar económico y social de una manera equitativa, y sin comprometer la sostenibilidad de los ecosistemas". Este concepto reconoce la multiplicidad de los objetivos a los que atiende este recurso y pone de manifiesto que la gestión del ciclo integral del agua debe descansar sobre tres criterios básicos: equidad social, eficiencia económica y sostenibilidad ecológica ${ }^{4}$. En esta línea, la

3 Global Water Partnership, 2000.

4 Idem, e lbidem, 2011. 
OECD subraya el importante papel que juegan los gobiernos a la hora de evitar los fallos de mercado asociados con este recurso, así como asegurar el cumplimiento de los principios anteriormente señalados y la adecuada distribución de los recursos hídricos entre los distintos usos a los que se puede atribuir ${ }^{5}$.

A la vista de las características particulares y condicionantes del sector, se podría aducir que la intervención del sector público en la industria del agua permitiría evitar los fallos de mercado y alcanzar un nivel óptimo de bienestar, constituyendo por tanto la provisión directa por parte de los gobiernos la forma más eficaz de prestación del servicio; de hecho, esta ha sido tradicionalmente la forma más habitual de titularidad del servicio. En los últimos años se viene observando, por el contrario, una tendencia creciente a la participación del sector privado en la provisión del servicio urbano de agua mediante distintas fórmulas, si bien su intensidad difiere de unos países a otros. La tabla 1 muestra el nivel de participación privada en los países desarrollados, medido en función del porcentaje de la población que es servida por la empresa privada.

Tabla 1. Nivel de participación del sector privado en la industria de distribución de agua en los países de la OECD (porcentaje de la población abastecido por el sector público)

\begin{tabular}{ll}
\hline $\begin{array}{l}\text { Bajo o inexistente } \\
\text { (menos del 10\%) }\end{array}$ & $\begin{array}{l}\text { Austria, Bélgica, Canadá, Corea del Sur, } \\
\text { Dinamarca, Finlandia, Irlanda, Islandia, } \\
\text { Japón, Luxemburgo, Noruega, Nueva } \\
\text { Zelanda, Países Bajos, Polonia, Suecia, } \\
\text { Suiza y Turquía. }\end{array}$ \\
\hline $\begin{array}{l}\text { Moderado } \\
\text { (entre 20\% y 30\%) }\end{array}$ & $\begin{array}{l}\text { Alemania, Australia, Hungría, México, } \\
\text { Portugal, Eslovaquia y Estados Unidos. }\end{array}$ \\
\hline $\begin{array}{l}\text { Alto } \\
\text { (entre 30\% y 50\%) }\end{array}$ & España, Grecia e Italia. \\
\hline
\end{tabular}

\begin{tabular}{ll}
\hline $\begin{array}{l}\text { Mayoritario } \\
\text { (entre 50\% y 70\%) }\end{array}$ & República Checa. \\
\hline $\begin{array}{l}\text { Predominante } \\
\text { (más del 70\%) }\end{array}$ & Francia e Inglaterra. \\
\hline
\end{tabular}

Fuente: Pérard, 2009.

Según la $O E C D^{6}$, la participación privada puede adquirir diversas formas en función de dónde recaiga la responsabilidad de los activos, las operaciones, la gestión, el riesgo y la inversión, así como en el estatus legal del operador del servicio. Dejando al margen la forma de propiedad, en el caso particular de la titularidad del gestor del servicio urbano de agua, se observan de forma genérica cuatro fórmulas principales. La administración pública sobre la que recae la responsabilidad de proveer el servicio puede optar por hacerlo "de forma directa o internamente", o bien externalizarlo. En caso de externalización, esta podría realizarse a través de una empresa de gestión "totalmente pública", una empresa de gestión "totalmente privada" (contractual public-

\footnotetext{
5 OECD, 2003.

6 Ibidem, 2008.
}

private partnership, en terminología anglosajona) o una empresa mixta público-privada (institutional public-private partnership).

La coexistencia de fórmulas tan diversas de participación del sector privado en el servicio urbano de aguas no hace más que evidenciar el hecho de que, a pesar de que existen desde el punto de vista teórico numerosos argumentos a favor y en contra de la privatización de los servicios de agua, la evidencia empírica al respecto no ha permitido arrojar luz de forma definitiva a la discusión acerca de qué forma de gestión se muestra superior desde la perspectiva de la eficiencia.

A continuación vamos a hacer un resumen de las distintas argumentaciones que desde el punto de vista teórico se han alegado a favor y en contra de la privatización en este sector.

\section{REVISIÓN TEÓRICA DE LOS ARGUMENTOS EN CONTRA \\ O A FAVOR DE LA PRIVATIZACIÓN}

\section{ARGUMENTOS A FAVOR}

Para comprender el origen de la privatización de los servicios urbanos de agua debemos remontarnos a los años ochenta del pasado siglo. Esta década fue testigo de un importante proceso de liberalización y desregulación de la economía en un gran número de países desarrollados para reducir la influencia del sector público en la actividad económica, con el fin último de promover la eficiencia y la consiguiente mejora en el bienestar social. El primer programa de privatización a gran escala fue el llevado a cabo por la administración Thatcher en el Reino Unido, aunque muy pronto las medidas implementadas ganaron una gran popularidad y su uso fue extendido a otros países. Este proceso privatizador se fundamentó principalmente en tres teorías que propugnaban una mayor eficiencia por parte del gestor privado: La Teoría de la Elección Pública, la Teoría de la Agencia y la Teoría de los Derechos de Propiedad.

La Teoría de la Elección Pública (Public Choice Theory) sostiene que el objetivo primordial de los políticos, lejos de perseguir un mayor bienestar social, es la maximización de su propio beneficio ${ }^{7}$. Esta afirmación tiene importantes consecuencias sobre la eficiencia de los servicios públicos provistos en calidad de monopolio. Por una parte, la búsqueda del interés propio por parte del gestor público conduciría a fallos de mercado como la excesiva provisión del servicio ${ }^{8}$; por otro lado, el carácter público del servicio impide la apropiación del beneficio generado por parte del gestor, lo que en un contexto de falta de competencia en precios, conllevaría un menor control sobre los costes de la organización, con la consecuente pérdida de eficiencia (Teoría de los Derechos de Propiedad o Property Rights Theory). En este escenario, la privatización de la gestión se contempla como una forma de introducir competencia y acabar con los problemas anteriormente mencionados, siempre y cuando el proceso de externalización se realice mediante procesos competitivos?.

\footnotetext{
7 Niskanen, 1971.

8 Ibidem. Bel et al., 2010.

9 Tiebout, 1956. Osborne y Gaebler, 1992.
} 
En esta misma línea, según la Teoría de la Agencia (Agency Theory $)^{10}$, la separación entre propiedad y control en las organizaciones conduce al denominado "problema principal-agente". Para el caso de los servicios públicos, este dilema se concreta en la existencia de un conflicto de intereses entre el ciudadano (principal) y el gestor público (agente), que conlleva una serie de costes, denominados "costes de agencia", derivados del acceso diferenciado a la información (asimetrías de la información). Según Jensen y Meckling ${ }^{11}$, existen una serie de mecanismos a través de los cuales el principal puede limitar esta desviación de sus intereses, entre los que figuran el establecimiento de incentivos que alineen los objetivos del agente con los suyos propios y la implementación de sistemas de monitorización de la actividad del agente. Sin embargo, en el caso de la empresa pública, la introducción de estos mecanismos se revela bastante más compleja que en la empresa privada. Una de las causas de ello radica en el marco laboral más restrictivo que afrontan este tipo de empresas y que impide el diseño de incentivos adecuados. Otra podría derivarse de la existencia de una doble relación principal-agente en las empresas públicas, que ocasionaría un incremento en los costes de control sobre el agente ${ }^{12}$.

Otro de los argumentos a los que habitualmente se hace referencia es la multiplicidad de objetivos a los que atiende la gestión pública frente al objetivo único de maximización del beneficio de la gestión privada. Desde el punto de vista de la eficiencia económica, se alega que la concurrencia de objetivos de naturaleza social, política y económica podrían conducir a una menor eficiencia de los servicios públicos de gestión del agua ${ }^{13}$.

A este respecto, una de las principales restricciones deriva de las impuestas por el entorno político en el que se enmarca la gestión. En relación a ello, se menciona el hecho de que a menudo la elección del gestor en un servicio de gestión pública dependerá más de sus características políticas que de su capacidad como gestor. Además, es lógico pensar que la toma de decisiones por parte de un gestor político estará fuertemente influenciada por la ideología a la que pertenezca (Teoría Partidista o Partisan Politics Theory) y se ha demostrado ampliamente la utilización oportunista por parte de estos de los instrumentos a su alcance con el fin de aumentar las probabilidades de ser reelegidos (Teoría de los Ciclos Políticos o Theory of Political Budget Cycles), factores ambos que actuarian en detrimento de la eficiencia.

También se hace referencia a una serie de factores de índole financiera para justificar la privatización de estos servicios (y como posibles causas de una menor eficiencia en el sector público frente al privado). Es común que los gobiernos locales afronten restricciones fiscales, ya sea por una elevada carga financiera, transferencias insuficientes por parte de los gobiernos centrales 0 en forma de leyes que impidan un incremento en el nivel impositivo o limiten el endeudamiento. En este orden de ideas, la privatización permite delegar en el gestor privado la acometida de inversiones de mejora y mantenimiento de la red, que de otro

\footnotetext{
10 Berle y Means, 1932.

11 Jensen y Meckling, 1976.

12 González-Gómez, 2006.

13 Martínez-Espiñeira et al., 2009.
}

modo habría que postergar. Además, permitiría la obtención de ingresos, tanto de forma inmediata mediante la venta como a través de una mayor recaudación futura por impuestos ${ }^{14}$.

Asimismo, se suele alegar que la existencia de relaciones laborales más rígidas supone un importante factor limitante de la eficiencia. En el caso de la empresa pública, existe una mayor dificultad para realizar ajustes de plantilla y el grado de sindicalización al que se enfrentan es mayor, factores ambos que limitan la flexibilidad y la capacidad adaptativa de la entidad, afectando negativamente a su eficiencia.

\section{ARGUMENTOS EN CONTRA}

Si bien los argumentos presentados en el epígrafe anterior permiten augurar que la privatización podría considerarse beneficiosa para la mejora de la eficiencia en la provisión del servicio urbano de aguas, existe también una abundante literatura acerca de sus efectos negativos sobre la misma. La privatización es contemplada frecuentemente como una vía para poner fin a las fuentes de ineficiencia generadas por la ausencia de competencia. Sin embargo, en numerosas ocasiones los efectos de la privatización sobre la competencia en el sector del servicio urbano de aguas son muy limitados ${ }^{15}$, de manera que la privatización por sí misma puede no ser la mejor forma de introducir competencia.

Algunos de los mecanismos habitualmente empleados para impulsar esta competencia se basan en conseguir un nivel adecuado de competencia por el mercado o mediante técnicas que atenúen el impacto de las asimetrías de la información, promoviendo la comparación entre unidades o la transparencia para una adecuada supervisión. La competencia por el mercado se genera principalmente a través de procesos de licitación. Lo ideal sería que a estos procesos de licitación del servicio público de agua acudieran un gran número de empresas fomentando así unas ofertas más competitivas. Sin embargo, su condición de monopolio local convierte a la industria del agua en un sector proclive a la concentración ${ }^{16}$, quedando en la práctica dominado por unas pocas empresas de gran tamaño. Es común, por tanto, que una sola empresa o un número muy reducido de ellas se presenten a los procesos de licitación, de forma que en la práctica el poder de esta herramienta a la hora de garantizar la defensa de los intereses públicos queda muy reducido.

Por su parte, la aplicación de técnicas de benchmarking puede fomentar la competencia por comparación en situaciones en las que la competencia dentro del propio mercado es escasa. Estas técnicas se basan en la medición del rendimiento de los operadores del servicio y su comparación, con el objetivo de incentivar a los mismos a introducir mecanismos correctivos que les permitan mejorar su eficiencia. No obstante, estas técnicas tampoco están exentas de obstáculos. En algunos países, el acceso a la información necesaria puede ser bastante dificultoso; asimismo, las fusiones 0 adquisiciones de unas compañías por parte de otras implican que en ocasiones no se cuente con suficientes observaciones independientes para llevar a cabo este tipo de análisis.

\footnotetext{
14 González-Gómez et al., 2011.

15 Hall y Lobina, 2004. Hall et al., 2005.

16 Sclar, 2000.
} 
Otro de los argumentos que se utiliza en contra del proceso privatizador se refiere a los elevados costes de transacción que conlleva. Estos costes de transacción incluyen tanto los costes de burocracia relacionados con la elaboración de contratos en los que se recojan pormenorizadamente las condiciones de prestación del servicio, como los costes incurridos como consecuencia de la monitorización y control del cumplimiento de las mismas. No debemos olvidar que la transacción ocurrirá solo cuando los beneficios generados por la misma sean mayores que sus $\operatorname{costes}^{17}$. En este orden de ideas, si los costes derivados de la transacción superasen las presuntas ganancias de eficiencia, la operación podría no ser deseable desde un punto de vista estrictamente económico.

Además, en el caso del servicio urbano de agua, la necesidad de realizar elevadas inversiones exige que el periodo de concesión del servicio sea suficientemente dilatado como para recuperar dichas inversiones. Por tanto, uno de los principales inconvenientes a los que se enfrenta la industria del agua es precisamente la práctica imposibilidad de redactar contratos que cubran todas las posibles contingencias y salvaguarden al mismo tiempo objetivos como el acceso universal al recurso 0 la sostenibilidad medioambiental, en un horizonte temporal tan amplio. Teniendo en cuenta que el objetivo primordial de la gestión privada reside únicamente en la maximización del beneficio, esta dificultad no haría más que reforzar el poder de mercado derivado de la situación de escasa competencia, dando lugar a una situación poco deseable desde la perspectiva de los intereses públicos. En relación con esta última circunstancia, la preocupación de los investigadores y poderes públicos se concentra principalmente, ante un proceso privatizador, en analizar el comportamiento que sufrirían tres de las principales variables, a saber, el precio del recurso, la calidad de prestación del servicio y la inversión en la mejora y mantenimiento de la red. Concretamente, gran número de estudios señalan la existencia de precios más elevados y menor calidad en el servicio, no justificados por factores ambientales, en los municipios provistos por gestores privados.

Respecto a la inversión en las redes de abastecimiento y saneamiento, algunos autores como Lobina y Hall ${ }^{18}$ sostienen que la inversión por parte de empresas de gestión pública se ha probado más eficaz tanto en países en desarrollo como en países desarrollados. Entre las ventajas derivadas de la financiación pública de estas inversiones destacarían los menores tipos de interés afrontados por el sector público con respecto a los inversores privados, la consecución de objetivos de carácter social como el acceso universal al recurso y los beneficios de salud pública ocasionados por la mejora de las redes. En relación a esto, las pérdidas de agua en la red son un problema que merece especial atención y mención por su particular importancia en un contexto de creciente estrés hídrico a nivel mundial. Es lógico pensar que la empresa privada no acometerá inversiones destinadas a evitar estas pérdidas si el coste de las mismas excede el ahorro por la pérdida de agua evitada. El hecho de que en la estructura de costes de la empresa no se contemplen los costes medioambientales y otras

\footnotetext{
17 Coase, 1937.

18 Lobina y Hall, 2000.
}

externalidades generadas por dicha decisión conduciría a un nivel de inversión inferior al que sería óptimo teniendo en cuenta todas estas circunstancias.

Dada la condición del agua de bien de mérito y necesidad básica humana, sería necesario evaluar de forma previa a la toma de decisión sobre la privatización si el incremento esperado en la eficiencia como consecuencia de dicha privatización compensa el coste social derivado de la pérdida de control sobre las tres variables anteriormente mencionadas.

\section{ANÁLISIS DE LA EVIDENCIA EMPÍRICA}

La ausencia de acuerdo a nivel teórico ha propiciado una extensa literatura empírica en torno a los efectos de la privatización sobre la eficiencia en el sector del agua. El interés de los investigadores en esta cuestión se remonta a los años setenta del pasado siglo con los artículos seminales de Mann y Mikesell ${ }^{19}$, Morgan ${ }^{20}$ y Crain y Zardkoohi ${ }^{21}$. Estos primeros estudios se realizaron para el caso de Estados Unidos y este país continuó siendo el principal objeto de estudio hasta mediados de los años noventa.

La privatización de la industria del agua en Inglaterra y Gales en noviembre de 1989 y la posterior creación de la OFWAT (Office of Water Services) para vigilar el cumplimiento del nuevo régimen regulatorio destinado a promover tanto la eficiencia económica en la industria como la calidad del recurso suministrado, provocó que la atención se desviara en la siguiente década hacia el Reino Unido. Este contexto proporcionó a los investigadores un doble experimento natural que permitía evaluar tanto los efectos que la privatización puede tener en la eficiencia como la efectividad de los nuevos mecanismos de regulación y supervisión introducidos. Uno de los estudios más destacados con relación a este doble objetivo fue el llevado a cabo por Saal y Parker ${ }^{22}$ con información sobre la industria del Reino Unido para el periodo 1985-1999, esto es, anterior y posterior a su privatización en 1989. Mediante la estimación de una función de costes, estos autores llegaron a la conclusión de que la privatización por sí misma no supuso una mejora de la eficiencia en la industria en este periodo, lo que constituía uno de los principales objetivos perseguidos con la reforma. Resultados similares fueron obtenidos por $\operatorname{Ashton}^{23} 0$ Hunt y Lynk ${ }^{24}$ para el caso anglosajón.

Desde entonces, el interés de los investigadores en esta cuestión ha sido creciente. Si bien hacia finales de los ochenta los artículos publicados no superaban la treintena, solo en la década de los noventa se publicaron alrededor de cuarenta investigaciones adicionales y para 2010, el número de publicaciones sobre eficiencia en el sector del agua superaba las $250^{25}$. Además, en la actualidad el debate se ha extendido a otros países y es posible encontrar investigaciones procedentes de los cinco continentes. Algunas de ellas centradas en países en desarrollo como Brasil,

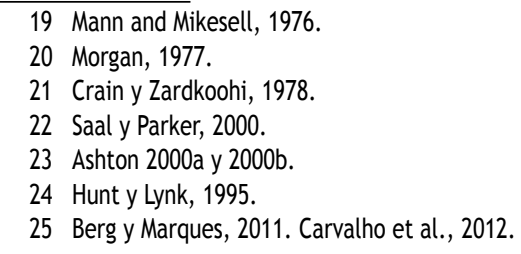


Argentina, México, Perú, Malasia o diversos países de África y de Asia y la región del Pacífico (Ver referencias en tabla 2). También es posible encontrar estudios a este respecto sobre países desarrollados como Alemania, España, Italia, Francia, Bélgica, Países Bajos, Portugal, Estonia o Australia.

Nuestro objetivo con este trabajo es realizar un análisis pormenorizado de la literatura más relevante hasta la fecha para proporcionar una perspectiva general de las evidencias aportadas por los estudios empíricos. En primer lugar, nos centraremos en examinar las distintas aproximaciones metodológicas utilizadas por estos estudios, para posteriormente comentar los resultados que las principales investigaciones arrojan desde el punto de vista de la superioridad de unas formas de gestión frente a otras.

APRoximaciones metodológICAS PARA EL ANÁLISIS

DE LA EFICIENCIA EN LA LITERATURA EMPÍRICA

Con carácter general, las técnicas cuantitativas utilizadas para medir la eficiencia pueden dividirse en paramétricas y no paramétricas, en función de que se asuma o no la existencia de una relación funcional para las funciones o fronteras de beneficios, costes y producción. En las técnicas paramétricas se establece una especificación funcional predeterminada y sus parámetros son estimados, habitualmente, a través de diversas técnicas econométricas. Dentro de esta metodología podemos dividir entre las que se basan en el uso de fronteras y las que no. En el primer caso, se define una frontera eficiente que representa las mejores prácticas y las desviaciones con respecto a la misma constituyen la medida de la ineficiencia; destaca en esta categoría el "análisis de fronteras estocásticas" o SFA (Stochastic Frontier Analysis) por sus siglas en inglés, en el que el calificativo "estocástico" hace referencia a la separación dentro del término de error de la parte debida a la ineficiencia y aquella que se debe a circunstancias no controlables por la unidad estudiada (ruido o parte puramente aleatoria). Entre aquellas técnicas paramétricas que no se basan en la utilización de fronteras destaca el análisis de regresión mediante funciones de producción o de costes.

Las técnicas no paramétricas, por su parte, no especifican ninguna forma funcional para la frontera que es estimada a partir de datos observados y un conjunto de axiomas sobre la tecnología. La técnica de análisis más habitual en esta categoría es el análisis envolvente de datos (DEA, Data Envelopment Analysis). En este caso, la frontera tecnológica que representa las mejores prácticas se construye utilizando herramientas de programación matemática y se obtienen índices de eficiencia comparando la situación de cada unidad con respecto a su equivalente en la frontera; dada la naturaleza multiproducto del sector del agua, esta técnica proporciona una ventaja adicional, la posibilidad de considerar de forma simultánea los outputs procedentes de las distintas etapas del ciclo integral del agua y los diversos inputs utilizados.

Con independencia de la técnica utilizada, las medidas de eficiencia más empleadas son principalmente dos: eficiencia técnica y eficiencia asignativa. La eficiencia técnica se refiere a la relación entre las cantidades físicas de inputs utilizadas y el nivel de output obtenido; en este sentido, una empresa es eficiente desde el punto de vista técnico cuando es capaz de obtener el máximo output dada la combinación y cantidad de inputs empleada (medida orientada al output) o utiliza la mínima cantidad de inputs necesaria para obtener su nivel de output (medida orientada al input). La eficiencia asignativa, por su parte, tiene en cuenta además los precios de los factores, ofreciendo una medición de la capacidad de la empresa de combinar sus inputs en proporciones que minimicen el coste de producción.

Sobre la base de la revisión efectuada, podemos concluir que la mayoría de las publicaciones sobre la industria del agua miden eficiencia técnica; en concreto, 26 artículos, frente a 15 que miden la eficiencia asignativa y 2 que miden ambas. La Tabla 2 sintetiza estos trabajos, incluyendo información relativa a la aproximación metodológica y medida de la eficiencia utilizada. Desde los artículos seminales de Mann y Mikesell ${ }^{26}$, Morgan ${ }^{27}$ y Crain y Zardkoohi ${ }^{28}$ hasta los primeros años del siglo XXI, se observa un predominio de las técnicas paramétricas, con alguna tentativa por parte de los investigadores de utilizar técnicas no paramétricas $^{29}$. Se distinguen en este periodo dos etapas. La mayoría de los estudios hasta mediados de los 90 se basaban en la estimación de funciones de $\operatorname{coste}^{30} 0$ de producción ${ }^{31}$, principalmente del tipo Cobb-Douglas o translogarítmica, mientras que a partir de entonces se impone el uso de fronteras, en un primer momento paramétricas (SFA) y en la actualidad la gran mayoría de los estudios se basan en la estimación de fronteras no paramétricas e indicadores de eficiencia mediante Análisis Envolvente de Datos. Sólo algunos estudios emplean otras técnicas no paramétricas. Tal es el caso de Byrnes et al. ${ }^{32}$, Saal y Parker ${ }^{33}$ o Estache y Trujillo ${ }^{34}$.

El análisis envolvente de datos (DEA) cuenta con diversas alternativas para definir los problemas de programación lineal, que podrían clasificarse en (i) orientados al input y al output; (ii) con Rendimientos Constantes de Escala (CRS, Constant Returns to Scale) o Rendimientos Variables de Escala (VRS, Variable Returns to Scale). Entre los artículos que componen nuestra revisión, la mayoría están orientados al input, es decir los indicadores de eficiencia proporcionan información sobre la reducción máxima de inputs que se podría llevar a cabo sin afectar al nivel de output producido por la empresa. Esta elección responde al hecho de que el objetivo deseado en este sector no es tanto aumentar la cuota de mercado y el nivel de consumo, puesto que dadas las características del sector de demanda suele estar determinada, como reducir los recursos utilizados para conseguir un determinado nivel de producción ${ }^{35}$. Sólo algunos de los estudios revisados utilizan la aproximación orientada al output ${ }^{36}$.

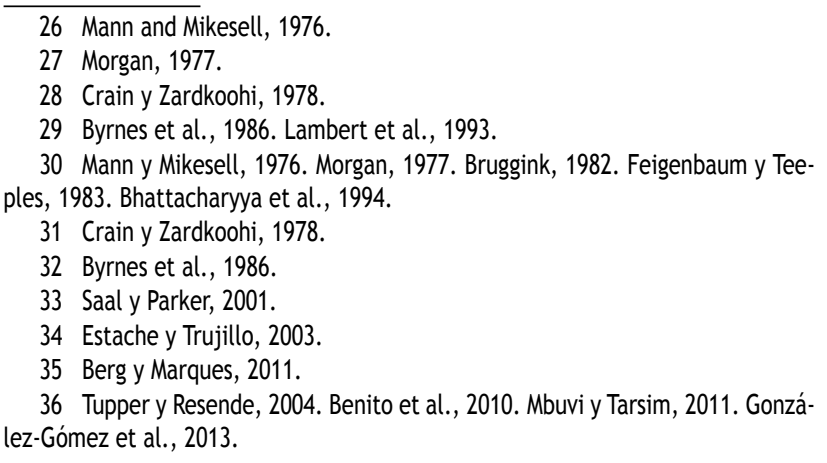




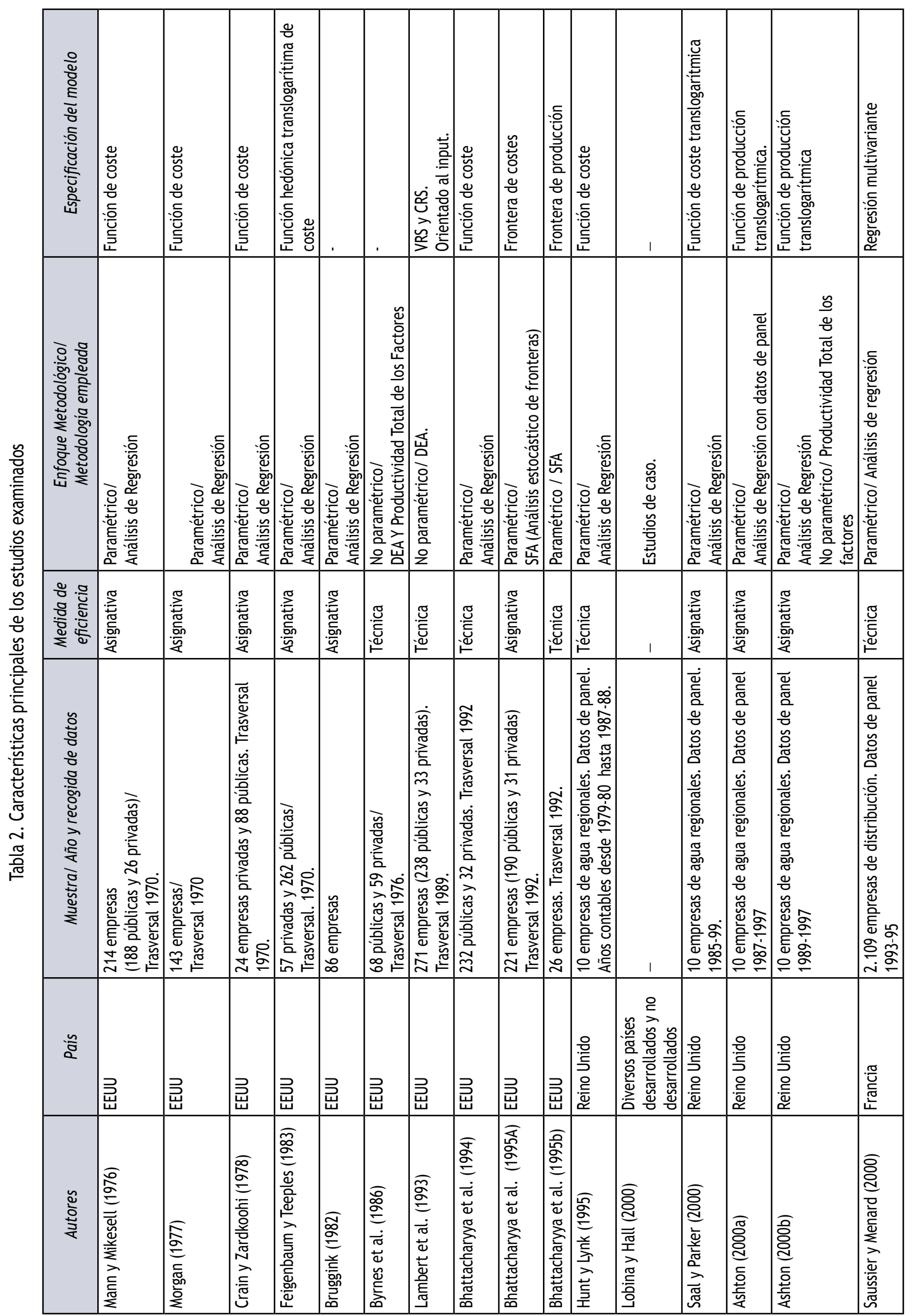




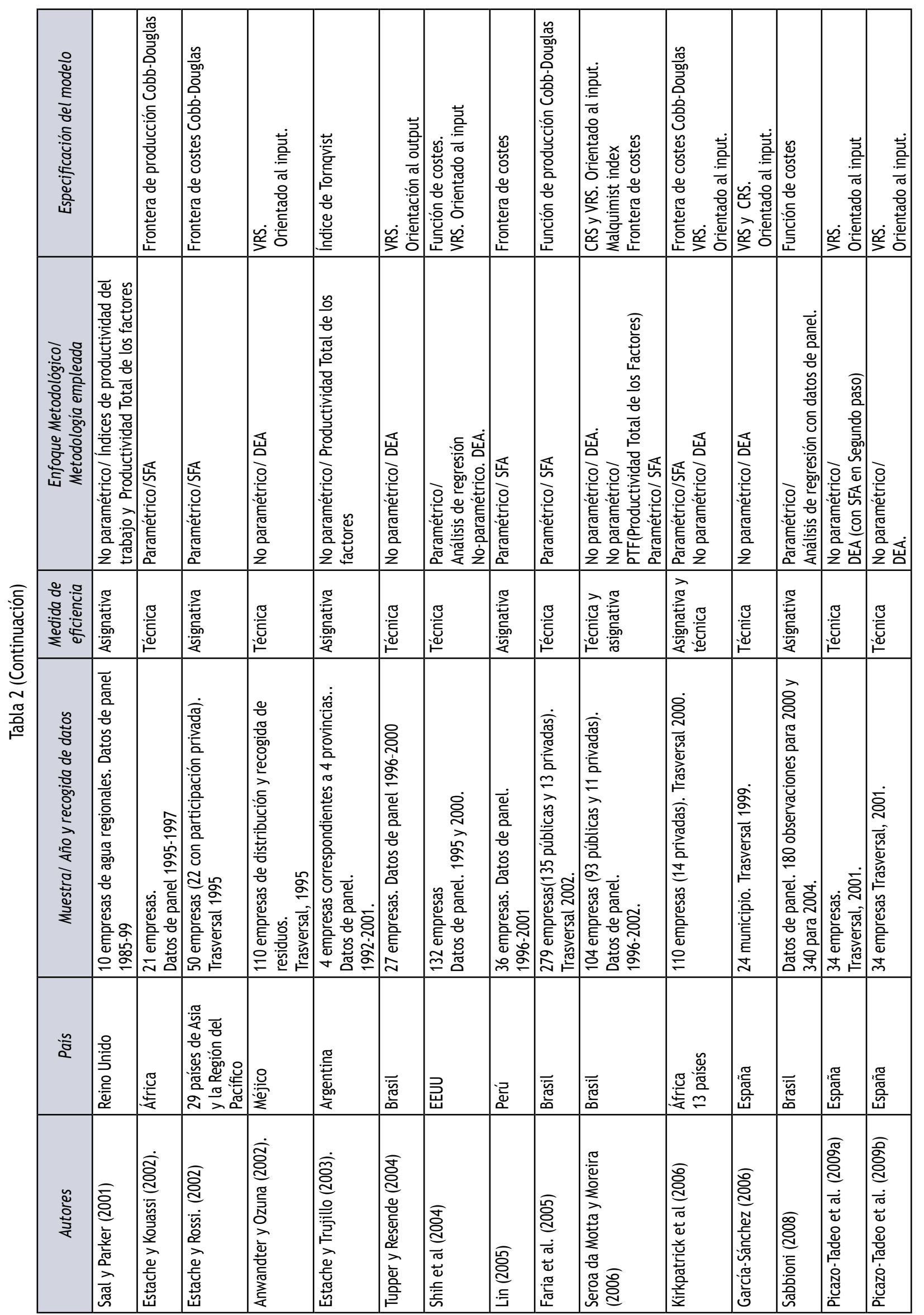




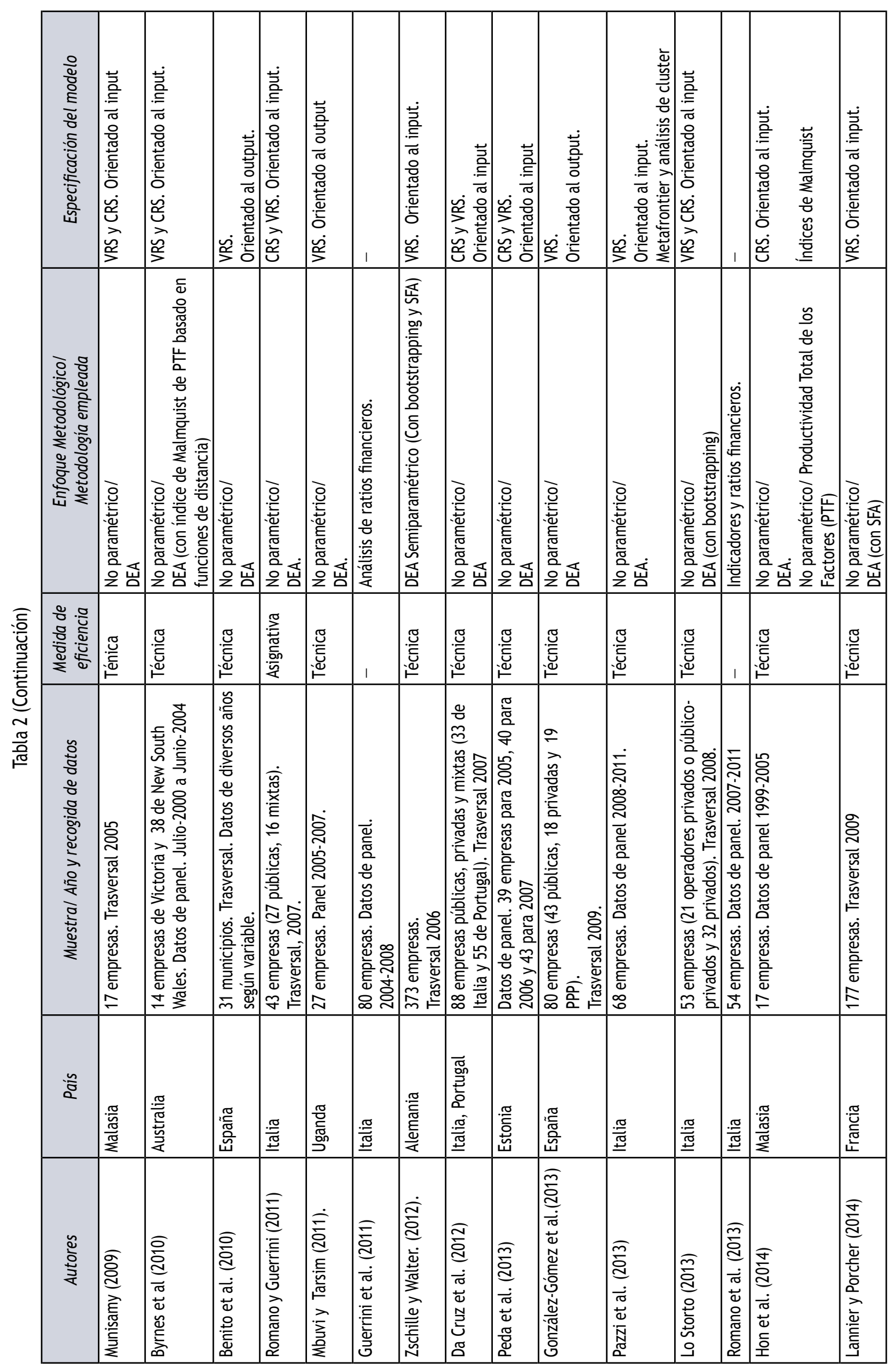

116 AGUA Y TERRITORIO, NÚM. 6, pp. 108-122, JULIO-DICIEMBRE 2015, ISSN 2340-8472, ISSN e 2340-7743, DOI 10.17561/at.vOi6.2814 
Con respecto a los rendimientos de escala, la mayoría de los estudios utilizan VRS (11 publicaciones), 9 presentan resultados obtenidos con los supuestos de VRS y CRS, mientras que solo una de las investigaciones consultadas utiliza DEA solo con CRS. La aproximación en la que se calculan las dos alternativas presenta la ventaja de que permite obtener qué parte de la ineficiencia de la empresa se debe a que el tamaño de la misma no se corresponde con su escala óptima (Ineficiencia de escala).

En cuanto al enfoque metodológico utilizado para medir qué tipo de gestión se muestra superior, la pública o la privada, lo más habitual es o bien llevar a cabo una regresión de los indicadores de eficiencia utilizados incluyendo variables dicotómicas que representen el tipo de gestión o utilizar contrastes de hipótesis que permitan concluir si las diferencias en eficiencia resultan o no significativas.

Respecto a la muestra, excepto en el caso de países de gran extensión y población como EEUU y Brasil, en los que la muestra puede llegar a sobrepasar las 200 empresas, en general el tamaño de muestra utilizado es pequeño. Esto se debe principalmente a que en ocasiones el número de empresas que operan en una determinada zona o país no es muy elevado y en otras responde a la dificultad para encontrar información sobre las variables necesarias para el análisis. En cuanto a la distribución temporal de la muestra, el número de estudios que utilizan datos de corte trasversal y datos de panel es bastante similar (22 trasversal frente a 21 que utilizan datos de panel). La estimación con datos de panel ofrece algunas ventajas ${ }^{37}$. Además de permitirnos controlar la heterogeneidad inobservable y mejorar la eficiencia de la estimación al disponer de más observaciones, para el caso concreto de los análisis de eficiencia esta estructura de los datos permite analizar los cambios producidos en la eficiencia a lo largo del tiempo 0 antes y después de un hecho puntual, que en el caso que nos ocupa podría tratarse de la privatización del servicio (o la municipalización, si bien no hay estudios empíricos que lo contemplen).

La elección del conjunto de inputs y outputs es otra de las decisiones que debe tomar el investigador en el momento de implementar el análisis de eficiencia. Entre los inputs más utilizados encontramos el factor trabajo, capital, la energía, el agua y materiales o consumos intermedios. La forma de introducir en el modelo estos factores de producción difiere sin embargo entre estudios. Para el caso del factor trabajo, lo ideal sería utilizar las horas de trabajo, incluyendo un coeficiente corrector del grado de cualificación $^{38}$. No obstante, en los estudios consultados, la variable proxy más habitual de este factor es el número de trabajadores ${ }^{39}$, en ocasiones diferenciando entre trabajadores a tiempo parcial y a tiempo completo ${ }^{40} 0$ las horas de trabajo totales en caso de encontrarse este dato disponible ${ }^{41}$. Entre las variables utilizadas para aproximar el factor capital, destaca la longitud de las redes de distribución, aunque a veces también el stock de capital ${ }^{42} 0$ el nú-

\footnotetext{
37 Schmidt y Sickles, 1984.

38 Estache et al., 2004. De Witte y Marques, 2010.

39 Estache y Trujillo, 2003. Kirkpatrick et al, 2006. García-Sánchez, 2006. Picazo-Tadeo et al., 2009a, 2009b.

40 Byrnes et al., 1986.

41 Lambert et al., 1993. Bhattacharyya et al., 1995b. Estache y Kouassi,

42 Bhattacharyya et al., 1995a.
} 2002. mero de conexiones ${ }^{43}$. El agua también es incluida como factor de producción en algunos de los estudios, distinguiendo en ocasiones entre el origen del recurso con el propósito de tener en cuenta las diferencias existentes en el coste de captación del mismo en función de su procedencia ${ }^{44}$. Para la energía, es frecuente incluir el número de $\mathrm{kw}$-hora consumidos ${ }^{45}$. Finalmente, cabe destacar que muchos estudios utilizan los costes como proxies de las cantidades físicas, en ausencia de información sobre estas últimas. A este respecto el más utilizado es los costes operativos ${ }^{46}$, en la medida en la que representa el coste soportado por la empresa como consecuencia de los distintos factores utilizados. Asimismo, es posible encontrar investigaciones que utilizan los costes de cada uno de los factores de forma separada ${ }^{47}$. Es destacable también, aunque menos frecuente, la utilización en años recientes de los ingresos totales como aproximación de los costes totales ${ }^{48}$, sobre todo en investigaciones realizadas con países europeos como caso de estudio, asumiendo que bajo el principio de recuperación de costes establecido en la Directiva Marco del Agua, los ingresos deberán cubrir dichos costes.

Con respecto al output, debemos tener en cuenta que la industria del agua es una industria multiproducto, que se encarga de la realización de las distintas etapas del ciclo integral del agua. Muchos de los estudios analizados tienen en cuenta esta circunstancia, incluyendo más de un output para considerar la diversidad de servicios ofrecidos por la industria ${ }^{49}$, aunque la mayoría de ellos se centran en un solo output. La etapa de distribución es sin duda la más tenida en cuenta en los estudios de eficiencia, seguida por la recogida y tratamiento de aguas residuales. La variable más utilizada para representar los servicios de distribución de agua es el volumen de agua distribuida ${ }^{50}$, aunque también se utiliza la población servida ${ }^{51} 0$ el número de conexiones a la red de distribución ${ }^{52}$.

En cuanto a los servicios de recogida y tratamiento de aguas residuales la variable más utilizada es el volumen de aguas residuales ${ }^{53}$, el agua residual tratada ${ }^{54} 0$ la población conectada a las plantas de tratamiento y recogida de residuos ${ }^{55}$.

\footnotetext{
43 Morgan, 1977.
}

44 Mann y Mikesell, 1976. Morgan, 1977. Byrnes et al., 1986. Anwandter y Ozuna, 2002. Picazo-Tadeo et al., 2009b.

45 Bhattacharyya et al., 1995b. Anwandter y Ozuna, 2002.

46 Estache y Rossi, 2002. Kirkpatrick et al., 2006. Picazo-Tadeo et al., 2009a. Munisamy, 2009. Byrnes et al. 2010. González-Gómez et al., 2013. Hon et al., 2014.

47 Bhattacharyya et al, 1995a y 1995b. Ashton, 2000a. Tupper y Resende, 2004. Da Cruz et al., 2012. Pazzi et al., 2013.

48 Zschille y Walter, 2012. Pazzi et al., 2013. Lo Storto, 2013.

49 Hunt y Lynk, 1995. Saal y Parker, 2001. Estache y Trujillo, 2003. Seroa da Motta y Moreira, 2006. Picazo-Tadeo et al., 2009a y $2009 \mathrm{~b}$.

50 Saal y Parker, 2001. Estache y Trujillo, 2003. Seroa da Motta y Moreira, 2006. Kirkpatrick et al., 2006. Sabioni, 2008. Picazo-Tadeo et al., 2009a. Byrnes et al., 2010. Zschille y Walter, 2012. Lannier y Porcher, 2014.

51 Saal y Parker, 2000. Mbuvi y Tarsim, 2011. Da Cruz et al., 2012. Peda et al. 2013.

52 Ashton, 2000a y 2000b. Estache y Rossi, 2002. García-Sánchez, 2006. Sabioni, 2008. Munisamy, 2009. Hon et al., 2014.

53 Saal y Parker, 2001. Estache y Trujillo, 2003. Seroa da Motta y Moreira, 2006. Picazo-Tadeo et al., 2009a y 2009b.

54 Seroa da Motta y Moreira, 2006. Picazo-Tadeo et al., 2009a y 2009b. Peda et al. 2013. González-Gómez et al., 2013.

55 Saal y Parker, 2000. 
Tabla 3. Principales resultados de la literatura en relación con la superioridad en términos de eficiencia de las distintas formas de gestión

\begin{tabular}{lll}
\hline \multicolumn{1}{c}{ Superioridad de la gestión pública } & \multicolumn{1}{c}{ Superioridad de la gestión privada } & \multicolumn{1}{c}{ No existe diferencia o inconclusivo. } \\
\hline Mann y Mikesell (1976) & Morgan (1977) & Feigenbaum y Teeples (1983) \\
Bruggink (1982) & Crain y Zardkoohi (1978) & Byrnes et al. (1986) \\
Lambert et al. (1993) & Bhattacharyya et al. (1995b) & Saussier y Menard (2000) \\
Bhattacharyya et al. (1994) & Estache y Kouassi (2002) & Estache y Rossi. (2002) \\
Bhattacharyya et al. (1995A) & Faria et al. (2005) & Seroa da Motta y Moreira (2006) \\
Lobina y Hall (2000) & Picazo-Tadeo et al. (2009a) & Kirkpatrick et al. (2006) \\
Shih et al. (2004) & Picazo-Tadeo et al. (2009b) & García-Sánchez (2006) \\
Benito et al. (2010) & Lo Storto (2013) & Sabbioni (2008) \\
Romano y Guerrini (2011) & & Munisamy (2009) \\
Guerrini et al. (2011) & & Zschille y Walter (2012) \\
Mbuvi y Tarsim (2011) & & Peda et al. (2013) \\
Da Cruz et al. (2012) & & González-Gómez et al. (2013) \\
Romano et al. (2013) & & Pazzi et al. (2013) \\
Lannier y Porcher (2014) & & Hon et al. (2014) \\
\hline
\end{tabular}

Mención especial merece la calidad. Dada la naturaleza del recurso, la calidad del mismo puede ser un producto tan deseable como su cantidad. A pesar de la influencia que esta variable puede tener en la medición de la eficiencia en el servicio, escasos son los estudios que la consideran. Para aquellos que lo hacen su inclusión se ha llevado a cabo de formas muy variadas: mediante la variable agua no contabilizada ${ }^{56}$, teniendo en cuenta las pérdidas de agua en el sistema ${ }^{57}$, en función del nivel de tratamiento sanitario recibido ${ }^{58}$, el número de controles y análisis realizados ${ }^{59}$, aplicando un coeficiente corrector a las cantidades producidas ${ }^{60} 0$ considerando el número de horas al día que se presta el servicio ${ }^{61}$.

Para concluir, cabe mencionar que gran parte de los artículos analizados tienen en cuenta la existencia de factores ambientales que pueden estar condicionando la eficiencia de las empresas del sector. La inclusión de este tipo de variables es importante para evitar atribuir a la mala gestión, diferencias que pueden ser debidas a circunstancias del entorno. Entre los factores considerados destacan la densidad de población, el ingreso per capita, la estacionalidad de la demanda, el origen del agua captada y la calidad del recurso (en ocasiones esta se incluye como factor ambiental en lugar de como output).

\section{¿QUÉ FORMA DE GESTIÓN SE MUESTRA SUPERIOR?}

La literatura empírica sobre las diferencias de eficiencia en la gestión pública o privada del servicio urbano de agua puede dividirse principalmente en dos grandes bloques, a saber, los estudios de corte transversal en los que se comparan los indicadores de eficiencia del sector público y privado, y los que analizan la

\footnotetext{
56 Anwandter y Ozuna, 2002. Tupper y Resende, 2004. Kirkpatrick et al., 2006. Picazo-Tadeo et al., 2008.

57 Bhattacharyya et al., 1995a. Lannier y Porcher, 2014.

58 Saussier y Ménard, 2000.

59 García-Sánchez, 2006.

60 Saal y Parker, 2001.

61 Estache y Rossi, 2002. Kirkpatrick et al., 2006.
}

tendencia que sufren dichos indicadores tras la implementación de un proceso privatizador. Este último grupo está formado principalmente por las investigaciones conducidas a raíz del proceso de reforma y privatización masiva ocurrido en el sector del agua en el Reino Unido en el año 198962, y encuentran de forma mayoritaria que el proceso privatizador no ha supuesto una ganancia de eficiencia en el sector del agua. Existe una excepción a estos estudios. Estache y Trujillo (2003), utilizando información de empresas de cuatro provincias argentinas desde el año 1992 al 2001, obtuvieron que la privatización llevó a ganancias de aproximadamente un $2 \%$ anual en la Productividad Total de los Factores. Aunque los autores advierten de la existencia de limitaciones metodológicas derivadas del pequeño tamaño muestral que obligan a tomar de forma cautelosa los resultados obtenidos.

Dentro del primer grupo de publicaciones, las conclusiones son más diversas y es posible encontrar resultados en todos los sentidos. Entre los 35 estudios revisados, 14 establecen una superioridad de la eficiencia en la gestión pública, 8 en la privada y 15 concluyen que 0 bien no encuentran diferencias significativas o la evidencia no les permite alcanzar una conclusión robusta al respecto (ver la Tabla 3). Esta variedad de resultados evidencia la falta de consenso entre los expertos con respecto a qué forma de gestión, pública o privada, arroja resultados más satisfactorios desde el punto de vista de la eficiencia. Si bien cabe destacar que desde el año 2009, el número de estudios que concluyen la superioridad de la gestión pública es sustancialmente mayor a los que establecen una superioridad de la privada, lo que parece apoyar la tendencia hacia la remunicipalización en el servicio del agua comentada antes.

Los estudios que encuentran que la gestión pública es más eficiente que la privada aducen diversos factores explicativos. Mann y Mikesell ${ }^{63}$ hallan mayores costes en las empresas privadas, que atribuyen al pago de salarios más elevados en este sector.

62 Hunt y Lynk, 1995. Saal y Parker, 2000 y 2001. Ashton, 2000a y 2000b.

63 Mann y Mikesell, 1976. 
El argumento de menores costes en el sector público es común también a Bruggink ${ }^{64}$, Bhattacharyya et al. ${ }^{65}$ y Shih et al ${ }^{66}$. Estos últimos puntualizan que al dividir las empresas por tamaños se observa que para el caso concreto de las empresas pequeñas, la gestión privada resulta más eficiente que la pública.

Otros artículos llegan a la conclusión de que la gestión pública es superior a la privada tras una exhaustiva revisión de estudios de caso. En particular, Lobina y Hall ${ }^{67}$ estudiaron pormenorizadamente situaciones procedentes de gran diversidad de países de distintas características, llegando a la conclusión de que de forma general las empresas privadas no se muestran superiores a las públicas en términos de eficiencia. Sin embargo, las públicas son capaces de dar una respuesta mucho más eficaz a consideraciones sociales y de desarrollo.

Los estudios de los últimos años, por contra, se basan principalmente en indicadores de eficiencia, encontrando una superioridad en la gestión pública ${ }^{68}$.

Con respecto a los estudios que encuentran una superioridad de la gestión privada encontramos los que apuntan a razones de coste $^{69} 0$ la superioridad en indicadores de eficiencia basados en técnicas de benchmarking ${ }^{70}$. Algunos de estos estudios destacan que las diferencias en eficiencia se producen principalmente en la utilización del factor trabajo $0^{71}$.

El grupo más numeroso de estudios corresponde a aquellos que 0 bien no encuentran diferencias significativas entre ambas formas de gestión o la evidencia empírica no les permite llegar a una conclusión. En este sentido, Saussier y Menard ${ }^{72}$ hallan que una vez descontado el efecto de los factores ambientales sobre la eficiencia, las diferencias entre gestión pública y privada no son significativas; similares conclusiones son alcanzadas por González-Gómez et $\mathrm{al}^{73}$. Otros autores obtienen que aunque no existan diferencias significativas, los operadores privados se han desplazado de forma más veloz hacia la frontera tecnológica que los públicos, pudiendo predecir un posible mejor rendimiento de estos a largo plazo ${ }^{74}$.

Cabe destacar que, a la vista de la revisión realizada, es posible adivinar el cada vez más destacado papel ejercido por las formas mixtas de gestión y el creciente empeño de los investigadores en su estudio. La controversia existente tanto desde el punto de vista teórico como empírico acerca de la dicotomía público-privado y la imposibilidad de alcanzar una respuesta concluyente a cuál se muestra superior, ha auspiciado que muchos autores vean en esta fórmula de gestión un medio para aunar las fortalezas de la gestión pública y la privada.

\footnotetext{
64 Bruggink, 1982.

65 Bhattacharyya et al. 1995a.

66 Shih et al. 2004.

67 Lobina y Hall, 2000.

68 Benito et al. 2010. Romano y Guerrini, 2011. Guerrini et al. 2011. Mbuvi y

Tarsim, 2011. Da Cruz et al. 2012. Romano et al. 2013. Lannier y Porcher, 2014.

69 Morgan, 1977. Crain y Zardkoohi, 1978.

70 Estache y Kouassi, 2002. Picazo-Tadeo et al. 2009a y 2009b.

71 Crain y Zardkoohi, 1978. Picazo-Tadeo et al. 2009a y 2009b.

72 Saussier y Ménard, 2000

73 González-Gómez et al. 2013.

74 Seroa da Motta y Moreira, 2006.
}

Bajo forma de gestión mixta, el sector público participa en la toma de decisiones y establece objetivos relativos a los requisitos de prestación del servicio, mientras que se delega en el sector privado la gestión de las operaciones y, según las condiciones estipuladas, la financiación de las infraestructuras. Para las entidades locales, la colaboración público-privada (institutional public-private partnership) presenta muchas ventajas. Es habitual que estas corporaciones se enfrenten a restricciones que les impidan financiar mejoras en las redes de abastecimiento y saneamiento, lo que deriva en un problema de infrainversión y el correspondiente deterioro del servicio ${ }^{75}$. Las formas mixtas de gestión permiten delegar en un gestor privado la gestión de la compañía y la financiación de las instalaciones sin necesidad de incrementar el nivel de deuda. Al mismo tiempo, el mayor grado de especialización en la gestión privada y su mayor flexibilidad y capacidad de innovación podrían permitir, con una adecuada supervisión, alcanzar de manera simultánea un nivel de eficiencia más elevado y mejor calidad en la prestación del servicio. Asimismo, dada la presencia de la empresa pública en los órganos en los que se toman las decisiones, esto no se haría en detrimento de la pérdida de control sobre objetivos sociales, económicos y políticos, y permitiría reducir los costes asociados a los contratos incompletos y al control y monitorización de la actividad del gestor al ejercerse estos de forma interna. Sin embargo, tampoco esta fórmula de gestión está exenta de cierta controversia y algunos autores señalan que la gestión privada está expuesta a presiones contradictorias que pueden llegar a convertirla en "lo peor de ambos mundos"76.

Entre los estudios recientes analizados, a partir de 2010 la gran mayoría de ellos tienen en cuenta la gestión mixta a la hora de investigar los efectos de la titularidad de la gestión en la eficiencia ${ }^{77}$, lo que resulta muy ilustrativo del aumento en su importancia.

Algunas de las investigaciones, si bien consideran la importancia de esta forma de gestión y la incluyen en sus análisis descriptivos, no llevan a cabo un análisis más profundo sobre la cuestión que les permita alcanzar una conclusión con respecto a la gestión mixta. Esto puede ser consecuencia de la metodología utilizada en el campo de investigación para comparar gestión pública y privada, basada principalmente en la creación de variables dicotómicas y contrastes de medias. Es habitual al utilizar estas metodologías, incluir la gestión mixta en el mismo grupo que la privada de forma que los efectos de ambas se confunden y no es posible estudiar el impacto de la gestión mixta de forma aislada.

Entre los resultados obtenidos encontramos los de Romano et al. $^{78} 0$ Romano et al. ${ }^{79}$, que concluyen que las empresas mixtas obtienen más beneficios que las privadas, pero que estos son explicados por un nivel de inversión más bajo y niveles tarifarios

\footnotetext{
75 Lo Storto, 2013. FMI, 2004.

76 Eckel y Vining, 1985. Da Cruz y Marques, 2012.

77 Benito et al. 2010. Romano y Guerrini, 2011. Romano et al. 2013. Zschille y Walter, 2012. Da Cruz et al. 2012. Peda et al. 2013. González-Gómez et al. 2013. Pazzi et al. 2013. Lo Storto, 2013. Romano et al. 2013. Lannier y Porcher, 2014.

78 Romano y Guerrini, 2011.

79 Ibidem. 2013.
} 
más elevados. En esta línea, Da Cruz y Marques ${ }^{80}$, basándose en cuatro estudios de caso, señalan que en las formas de gestión y propiedad mixta terminan por prevalecer los objetivos del gestor privado de maximización del beneficio frente al interés común. Sin embargo, inciden en la existencia de mecanismos que permiten asegurar el equilibrio entre las presiones contradictorias de búsqueda de eficiencia y consecución de objetivos sociales y apuntan a la necesidad de ahondar en los factores que permitirían el éxito de estas estructuras de gestión.

\section{ConCLUSIONES}

El debate acerca de qué forma de titularidad de la gestión de los servicios de agua, pública o privada, se muestra superior, ha sido recurrente durante las últimas tres décadas. Si bien organismos como el Fondo Monetario Internacional, la OECD o el Banco Mundial han venido aconsejando históricamente la privatización como vía para la mejora de la eficiencia en la industria del agua, en la última década muchas son las voces que reclaman una reconsideración de la gestión pública ${ }^{81}$. De hecho, numerosos municipios han llevado a cabo recientemente una remunicipalización de los servicios urbanos de agua, como forma de atender mejor a los objetivos que se esperan de su gestión. Tal es el caso de ciudades como París, Buenos Aires o Berlin ${ }^{82}$.

En este artículo hemos ofrecido una revisión de la literatura existente a nivel teórico y empírico sobre qué forma de titularidad de los servicios de agua urbana resulta más adecuada desde la perspectiva de la eficiencia en la gestión. La revisión efectuada nos permite concluir que no hay evidencia irrefutable acerca de la superioridad de una forma de titularidad de la gestión, pública o privada, frente a la otra. Sin embargo, es posible a la vista de la misma detectar algunos desafíos a los que deberían enfrentarse los investigadores en un futuro próximo:

- De un lado, los resultados de la gestión de una unidad están a menudo condicionados por factores propios del entorno en el que se desarrolla y que pueden estar contaminando la medida de eficiencia. Si bien muchos de los artículos consultados tienen en cuenta estos factores, una gran parte de ellos no incluyen ningún tipo de mecanismo para aislar sus efectos.

- Además, las formas de gestión mixta están adquiriendo una creciente relevancia en el panorama de la gestión de los servicios urbanos de agua. Sin embargo, como hemos comentado anteriormente en el texto, la metodología utilizada en el campo de investigación para comparar los indicadores de eficiencia, basada en la mayoría de los casos en la creación de variables dicotómicas y contrastes de medias para diferenciar gestor público y privado, podría estar impidiendo que se estudie el impacto sobre la eficiencia de las formas de gestión mixta de manera aislada.

80 Da Cruz y Marques, 2012.

81 Lobina y Hall, 2000. Hall y Lobina, 2004.

82 Lannier y Porcher, 2014. Pigeon et al. 2012. Beveridge y Naumann, 2013.
- Con respecto a la calidad, escasos son los estudios que contemplan su importancia. Las mejoras de eficiencia pueden estar realizándose en ocasiones en detrimento de la calidad del servicio prestado, por lo que considerar esta como un output adicional nos puede ofrecer una medida más precisa acerca de cómo una unidad determinada está gestionando sus recursos productivos. Teniendo en cuenta que un servicio urbano de agua de calidad puede generar numerosas externalidades positivas, considerar este factor puede contribuir notablemente a determinar qué forma de titularidad es más deseable para la sociedad en su conjunto.

Finalmente, nuestra revisión pone de manifiesto otras cuestiones aún por resolver en la industria, como el fomento de la competencia por el mercado y dentro del mismo, el impacto de la regulación, el papel y relevancia de los organismos supervisores o el diseño de incentivos económicos para reducir las pérdidas de agua en el sistema. Desde el punto de vista del debate que nos ocupa, sería interesante estudiar el impacto diferencial que las distintas cuestiones mencionadas podrían tener sobre la eficiencia en la gestión pública y privada.

La consideración conjunta de todas estas recomendaciones permitiría diseñar una combinación óptima de medidas que permitiera una mejora sustancial de la eficiencia en los servicios de agua urbana y contribuyera de este modo a afrontar los retos derivados de la sostenibilidad de los recursos hídricos a nivel global.

\section{Bibliografía}

Anwandter, L. y Ozuna, T. 2002: "Can public sector reforms improve the efficiency of public water utilities?", en Environment and Development Economics, 7, 4, 687-700.

Ashton, J. K. 2000a. "Cost efficiency in the UK water and sewerage industry", en Applied Economics Letters, 7, 7, 455-458.

Ashton, J. K. 2000b: "Total Factor Productivity Growth and Technical Change in the Water and Sewerage Industry", en The Service Industries Journal, 20, 4, 121-130.

Bel, G., Fageda, X. and Warner, M. 2010: "Is Private Production of Public Services Cheaper Than Public Production? A Meta-Regression Analysis of Solid Waste and Water Services", en Journal of Policy Analysis and Management, 29, 3, 553-577.

Benito, B., Bastida, F. y García, J. 2010: "Explaining differences in efficiency: an application to Spanish municipalities", en Applied Economics, 42, 4, 515-528.

Berg, S. V. y Marques, R. C. 2011. "Quantitative Studies of Water and Sanitation Utilities: A benchmarking Literature Survey", en Water Policy, 13, 5, 591-606.

Berle, A. A., y Means, G. C. 1932: The Modern Corporation and Private Property. New York, The Macmillan Company.

Beveridge, R. y Naumann, M. 2013: "The Berlin Water Company -- From "Inevitable" Privatization to "Impossible" Remunicipalization", en Bernt, M., Grell, B., y Holm, A. (edits.): The Berlin Reader. A Compendium on Urban Change and Activism. Serie Urban Studies. Bielefeld, Transcript. 
Bhattacharyya, A., Parker, E. y Raffiee, K. 1994: “An Examination of the Effect of Ownership on the Relative Efficiency of Public and Private Water Utilities", en Land Economics, 70, 2, 197-209.

Bhattacharyya, A., Harris, T. R., Narayanan, R., Raffiee, K. 1995a: "Specification and estimation of the effect of ownership on the economic efficiency of the water utilities", en Regional Science and Urban Economics, 25, 759-784.

Bhattacharyya, A., Harris, T. R., Narayanan, R., y Raffiee, K. 1995b: “Technical Efficiency of Rural Water Utilities", en Journal of Agricultural and Resource Economics, 20, 2, 373-391.

Bruggink, T. H. 1982: "Public Versus Regulated Private Enterprise in the Municipal Water Industry: A Comparison of Operating Costs", en Quarterly Review of Economics and Business, 22, 111-125.

Byrnes, P., Grosskopf, S. y Hayes, K. 1986: "Efficiency and Ownership: Further Evidence", en Review of Economics and Statistics, 68, 337341.

Byrnes, J., Crase, L., Dollery, B. y Villano, R. 2010. "The relative economic efficiency of urban water utilities in regional New South Wales and Victoria", en Resource and Energy Economics, 32, 3, 439-455.

Carvalho, P., Marques, R. C. y Berg, S. 2012: “A meta-regression analysis of benchmarking studies on water utilities market structure", en Utilities Policy, 21, 40-49

Coase, R. H. 1937: “The Nature of the Firm”, en Economica, 4, 386-405.

Crain, W. M. y Zardkoohi, A. 1978: "A test of the property-rights theory of the firm: Water utilities in the United States", en Journal of Law and Economics, 21, 2, 395-408.

Da Cruz, N. F. y Marques, R. C. 2012: "Mixed Companies And Local Governance: No Man Can Serve Two Masters", en Public Administration, 90, 3, 737-758.

Da Cruz, N. F., Marques, R. C., Romano, G. y Guerrini, A. 2012: “Measuring the efficiency of water utilities: a crossnational comparison between Portugal and Italy", en Water Policy, 14, 5, 841-853.

De Witte, K. y Marques, R. C. 2010: "Designing performance incentives: an International benchmark study in the water sector", en Central European Journal of Operations Research, 18, 2, 189-220.

Eckel, C. y Vining, A. 1985: "Elements of a Theory of Mixed Enterprise", en Scottish Journal of Political Economy, 32, 1, 82-94.

Estache, A. y Kouassi, E. 2002: "Sector Organization, Governance and The Inefficiency Of African Water Utilities", en World Bank, Policy Research Working Papers 2890. Washington, D. C.

Estache, A. y Rossi, M. A. 2002: "How Different is the Efficiency of Public and Private Water Companies in Asia?", en The World Bank Economic Review, 16, 1, 139-148.

Estache, A. y Trujillo, L. 2003: "Efficiency Effects of 'Privatization' in Argentina's Water and Sanitation Services", en Water Policy, 5, 4, 369-380.

Estache, A., Rossi, M. A. y Ruzzier, C. A. 2004: “The Case for International Coordination of Electricity Regulation: Evidence from the Measurement of Efficiency in South America", en Journal of Regulatory Economics, 25, 3, 271-295.

Faria, R. C., Souza, G. S., Moreira, T. B. S. 2005: "Public Versus Private Water Utilities. Empirical Evidence for Brazilian Companies", en Economics Bulletin, 8, 2, 1-7.

Feigenbaum, S. y Teeples, R. 1983: "Public Versus Private Water Delivery: A Hedonic Cost Approach", en Review of Economics and Statistics, 65, 4, 672-678.
Fondo Monetario Internacional, 2004: Public-Private Partnerships. Washington, Fiscal Affairs Department. International Monetary Fund.

García-Sánchez, I. M. 2006. "Efficiency Measurement in Spanish Local Government: The Case of Municipal Water Services, en Review of Policy Research, 23, 2, 355-372.

Global Water Partnership, 2000: Integrated Water Resources Management. TAC Background Papers N. 4. Stockholm, GWP Secretariat.

González-Gómez, F. 2006: “¿Está justificada la privatización de la gestión del agua en las ciudades? Teoría y evidencia a partir del criterio de eficiencia", en Ciudad y territorio. Estudios territoriales, 38, 147, 139-157.

González-Gómez, F., Picazo-Tadeo, A. J., y Guardiola, J. 2011: “Why do local governments privatize the provision of water services? Empirical evidence from Spain", en Public Administration, 89, 2, 471-492.

González-Gómez F. J., García-Rubio, M. A., Alcalá-Olid, F. y Ortega Díaz, M. I., 2013: "Outsourcing and Efficiency in the Management of Rural Water Services", en Water Resources Management, 27, 3, 731-747.

Guerrini, A., Romano, G. y Campedelli, B. 2011: "Factors affecting the performance of water utility companies", en International Journal of Public Sector Management, 24, 6, 543-566.

Hall, D. y Lobina, E. 2004: "Private and public interests in water and energy", en Natural Resources Forum, 28, 4, 268-277.

Hall, D., Lobina, E. y De la Motte, R. 2005: "Public resistance to privatization in water and energy", en Development in Practice, 15, 3-4, 286-301.

Hon, L. Y., Boon, T. H. y Lee, C. 2014: "Productivity, Efficiency and Privatization in the Malaysian Water Industry", en Journal of Southeast Asian Economies, 31, 2, 292-312.

Hunt, L. C. y Lynk, E. L. 1995: "Privatisation and Efficiency in the UK Water Industry: An Empirical Analysis", en Oxford Bulletin of Economics and Statistics, 57, 3, 371-388.

Jensen, M. C. y Meckling, W. H. 1976: "Theory of the Firm: Managerial Behavior, Agency Costs and Ownership Structure", en Journal of Financial Economics, 3, 4, 305-360.

Kirkpatrick, C., Parker, D. and Zhang, Y. F. 2006: "An Empirical Analysis of State and Private-Sector Provision of Water Services in Africa", en The World Bank Economic Review, 20, 1, 143-163.

Lambert, D. K., Dichev, D. y Raffiee, K. 1993: "Ownership and Sources of Inefficiency in the Provision of Water Services", en Water Resources Research, 29, 6,1573-1578.

Lannier, A. y Porcher, S. 2014: "Efficiency in the Public and Private French Water Utilities: Prospects for Benchmarking", en Applied Economics, 46, 5, 556-572.

Lo Storto, C. 2013: "Are Public-Private Partnerships a Source of Greater Efficiency in Water Supply? Results of a Non-Parametric Performance Analysis Relating to the Italian Industry", en Water, 5, 4, 20582079.

Lobina, E. and Hall, D. 2000: "Public Sector Alternatives to Water Supply and Sewerage Privatization. Case Studies", en International Journal of Water Resources Development, 16, 1, 35-55.

Mann, P. C. y Mikesell, J. L. 1976: "Ownership and water system operation", en JAWRA. Journal of the American Water Resources Association, 12, 5, 995-1004.

Martínez-Espiñeira, R., García-Valiñas, M., y González-Gómez, F. 2009: "Does Private Management of Water Supply Services Really Increase Prices? An Empirical Analysis in Spain”, en Urban Studies, 46, 4, 923-945. 
Mbuvi, D. y Tarsim, A. 2011: Managerial ownership and urban water utilities efficiency in Uganda. UNU-MERIT, Working Papers. Maastricht Economic and Social Research and Training Centre on Innovation and Technology.

Morgan, W. D. 1977: "Investor Owned vs. Publicly Owned Water Agencies: An Evaluation of the Property Rights Theory of the Firm", en JAWRA. Journal of the American Water Resources Association, 13, 4, 775-781.

Munisamy, S. 2009: "Efficiency and Ownership in Water Supply. Evidence from Malaysia", en International Review of Business Research Papers, 5, 6, 248-260.

Niskanen, W. A. 1971: Bureaucracy and representative government. Chicago, Aldine.

OECD, 2003: Social Issues in the Provision and Pricing of Water Services. Paris, OECD Publishing.

OECD, 2008: OECD. Environmental Outlook to 2030. Paris, OECD Publishing.

Osborne, D., y Gaebler, T. 1992: Reinventing Government: How the Entrepreneurial Spirit is Transforming the Public Sector. Reading, MA, Addison-Wesley.

Pazzi, S., Duygun, M., Tortosa-Ausina, E. y Zambelli, S. 2013: "Efficiency of Water Utilities: Does Local Public Ownership Matter? Evidence from Italy" Available at: https://editorialexpress.com/cgi-bin/conference/download.cgi?db_name=XXIEEP\&paper_id=98

Peda, P., Grossi, G. y Liik, M. 2013: "Do ownership and size affect the performance of water utilities? Evidence from Estonian municipalities", en Journal of Management and Governance, 17, 2, 237-259.

Pérard, E. 2009: "Water supply: Public or private? An approach based on cost of funds, transaction costs, efficiency and political costs", en Policy and Society, 27, 3,193-219.

Picazo-Tadeo, A. J., Sáez Fernández, F. J. y González Gómez, F. 2008: "Does service quality matter in measuring the performance of water utilities?", en Utilities Policy, 16,1, 30-38.

Picazo-Tadeo, A. J., González-Gómez, F. y Sáez-Fernández, F. J. 2009a: "'Accounting for Operating Environments in Measuring Water Utilities' Managerial Efficiency", en The Service Industries Journal, 29, 6, 761-773.

Picazo-Tadeo, A. J., Sáez-Fernández, F. J. y González Gómez, F. 2009b: "The role of environmental factors in water utilities' technical effi- ciency. Empirical evidence from Spanish companies", en Applied Economics, 41, 5, 615-628.

Pigeon, M., McDonald, D. A., Hoedeman, O. y Kishimoto, S. 2012: Remunicipalisation: Putting Water Back into Public Hands. Amsterdam, Transnational Institute.

Romano, G. y Guerrini, A. 2011: "Measuring and comparing the efficiency of water utility companies: A data envelopment analysis approach", en Utilities Policy, 19, 3, 202-209.

Romano, G., Guerrini, A. y Vernizzi, S. 2013: "Ownership, Investment Policies and Funding Choices of Italian Water Utilities: An Empirical Analysis", en Water Resources Management, 27, 9, 3409-3419.

Saal, D. S. y Parker, D. 2000: "The impact of privatization and regulation on the water and sewerage industry in England and Wales: a translog cost function model", en Managerial and Decision Economics, $21,6,253-268$.

Saal, D. S. y Parker, D. 2001: "Productivity and Price Performance in the Privatized Water and Sewerage Companies of England and Wales", en Journal of Regulatory Economics, 20, 1, 61-90.

Sabbioni, G. 2008: "Efficiency in the Brazilian sanitation sector", en Utilities Policy, 16, 1, 11-20.

Saussier, S. y Ménard, C. 2000: "Contractual Choice and Performance the Case of Water Supply in France", en Revue d'Économie Industrielle, 92, 1, 385-404.

Schmidt, P. y Sickles, R. 1984: "Production Frontiers and Panel Data", en Journal of Business and Economic Statistics, 2, 4, 367-374.

Sclar, E. D. 2000: You Don't Always Get What You Pay For: The Economics of Privatization. Ithaca, NY, Cornell University Press.

Seroa da Motta, R. y Moreira, A. 2006: "Efficiency and regulation in the sanitation sector in Brazil", en Utilities Policy, 14, 3, 185-195.

Shih, J. S., Harrington, W., Pizer, W. A. y Gillingham, K. 2004: Economies of Scale and Technical Efficiency in Community Water Systems. Discussion Paper 04-15. Washington, D. C., Resources for the Future.

Tiebout, C. M. 1956: “A Pure Theory of Local Expenditures”, en The Journal of Political Economy, 64, 5, 416-424.

Tupper, H. C. y Resende, M. 2004: "Efficiency and Regulatory Issues in the Brazilian Water and Sewage Sector: An Empirical Study", en Utilities Policy, 12, 1, 29-40.

Zschille, M. y Walter, M. 2012: "The Performance of German Water Utilities: A (Semi)-Parametric Analysis", en Applied Economics, 44, 29, 3749-3764. 